\title{
HARDY UNCERTAINTY PRINCIPLE FOR LOW DIMENSIONAL NILPOTENT LIE GROUPS G 4 (III)
}

\author{
C. R. Bhatta \\ Central Department of Mathematics \\ Tribhuvan University, Kirtipur \\ Corresponding author: crbhatta@yahoo.com \\ Received 25 October, 2009; Revised 17 February, 2010
}

\begin{abstract}
An uncertainty principle due to Hardy for Fourier transform pairs on $\Re$ says that if the function $\mathrm{f}$ is "very rapidly decreasing", then the Fourier transform can not also be "very rapidly decreasing" unless $f$ is identically zero. In this paper we state and prove an analogue of Hardy's theorem for low dimensional nilpotent Lie groups $\mathrm{G}_{4}$.
\end{abstract}

Keywords and phrases: Uncertainty principle, Fourier transform pairs, very rapidly decreasing, Nilpotent Lie groups.

\section{INTRODUCTION}

It is a well-known simple fact that if a function $f$ on $\mathfrak{R}$ is compactly supported then its fourier transform $\hat{f}$ cannot also be compactly supported, unless $f=0$. More generally, we have the following principle in classical Fourier analysis. If the function $\mathrm{f}$ is "very rapidly decreasing" then the Fourier transform can not also be "very rapidly decreasing" unless $f$ is identically zero. The following result of Hardy makes the rather vague statement above precise.

Let $\mathrm{g}$ be an $\mathrm{n}$-dimensional real Nilpotent Lie algebra and $\mathrm{G}=\exp \mathrm{g}$ be the associated connected and simply connected Nilpotent Lie group. Let $\left\{\mathrm{x}_{1}, \ldots \mathrm{x}_{\mathrm{n}}\right\}$ be a strong Malcev basis of $g$ through the ascending central series of $\mathrm{g}$. In particular, $\mathfrak{R X _ { 1 }}$ is contained in the centre of $\mathrm{g}$. We introduce a norm function on $\mathrm{G}$ by setting for

$$
\mathrm{x}=\exp \left(\mathrm{x}_{1} \mathrm{X}_{1}+\ldots+\mathrm{x}_{\mathrm{n}} \mathrm{X}_{\mathrm{n}}\right) \in \mathrm{G}, \mathrm{x}_{\mathrm{j}} \in \mathfrak{R}
$$

The composed map

$$
\mathfrak{R}^{\mathrm{n}} \rightarrow \mathrm{g} \rightarrow \mathrm{G},\left(\mathrm{x}_{1}, \ldots, \mathrm{x}_{\mathrm{n}}\right) \rightarrow \sum_{\mathrm{j}=1}^{\mathrm{n}} \mathrm{x}_{\mathrm{i}} \mathrm{X}_{\mathrm{j}}
$$

is a diffeomophism and maps Lebesgue measure on $\Re^{\mathrm{n}}$ to Haar measure on G. In this manner, we shall always identify g and sometimes $G_{1}$ as sets with $\mathfrak{R}^{\mathrm{n}}$. The measurable (integrable) functions on $\mathrm{G}$ can be viewed as such functions on $\mathfrak{R}^{\mathrm{n}}$.

Let $g$ denote the vector space dual of $g$ and $\left\{X_{1}^{*}, \ldots, X_{n}^{*}\right\}$ the basis of $g^{*}$ which is dual to $\left\{\mathrm{X}_{1}, \ldots, \mathrm{X}_{\mathrm{n}}\right\}$. Then $\left\{\mathrm{X}_{1}^{*}, \ldots, \mathrm{X}_{\mathrm{n}}^{*}\right\}$ is Jordon Holder basis for the coadjoint action of $\mathrm{G}$ on $g^{*}$. We shall identify $g^{*}$ with $\mathfrak{R}^{\mathrm{n}}$ via the map $\xi=\left(\xi_{1}, \ldots, \xi_{\mathrm{n}}\right) \rightarrow \sum_{\mathrm{j}=1}^{\mathrm{n}} \xi_{\mathrm{j}} \mathrm{X}_{\mathrm{j}}^{*}$ and on $\mathrm{g}^{*}$. We introduce the Eucledian norm relative to the basis $\left\{\mathrm{X}_{1}^{*}, \ldots, \mathrm{X}_{\mathrm{n}}^{*}\right\}$, that is 


$$
\left\|\sum_{\mathrm{j}=1}^{\mathrm{n}} \xi_{\mathrm{j}} X_{\mathrm{j}}^{*}\right\|=\left(\xi_{1}^{2}+\xi_{2}^{2}+\ldots+\xi_{\mathrm{n}}^{2}\right)^{1 / 2}=\|\xi\| .
$$

For an operator $\mathrm{T}$ in a Hilbert space such that $\mathrm{T}^{*} \mathrm{~T}$ is a trace class. $\|\mathrm{T}\|_{\mathrm{HS}}$ will denote the Hilbert Schmidt norm of T.

\section{THREAD LIKE NILPOTENT LIE GROUPS}

For $\mathrm{n} \geq 3$, let $\mathrm{g}_{\mathrm{n}}$ be the $\mathrm{n}$-dimensional real Nilpotent Lie algebra with basis $\mathrm{X}_{1}, \ldots, \mathrm{X}_{\mathrm{n}}$ and non trivial lie brackets $\left[\mathrm{X}_{1}, \mathrm{X}_{\mathrm{n}-1}\right]=\mathrm{X}_{\mathrm{n}-2}, \ldots,\left[\mathrm{X}_{1}, \mathrm{X}_{2}\right]=\mathrm{X}_{1}$.

$\mathrm{g}_{\mathrm{n}}$ is a (n - 1) step Nilpotent and is a product of $\mathfrak{R} X_{n}$ and the abelian ideal $\sum_{j=1}^{n-1} \mathfrak{R} X_{j}$. Note that $g_{3}$ is the Heisenber Lie algebra. Let $G_{n}=\exp \left(g_{n}\right)$.

For $\xi=\sum_{j=1}^{n-1} \xi_{1} X_{j}^{*} \in g_{n}^{*}$, the coadjoint action of $G_{n}$ is given by

$$
\operatorname{Ad}^{*}\left(\exp \left(t X_{n}\right) \xi=\sum_{j=1}^{n-1} P_{j}(\xi, t) X_{j}^{*}\right.
$$

where for $\mathrm{i} \leq \mathrm{j} \mathrm{n}-1, \mathrm{P}_{\mathrm{j}}(\xi, \mathrm{t})$ is the polynomial in $\mathrm{t}$ defined by

$$
\mathrm{P}_{\mathrm{j}}(\xi, \mathrm{t})=\sum_{\mathrm{k}=1}^{\mathrm{j}-1}(1 / \mathrm{k} !)(-1)^{\mathrm{k}} \mathrm{t}^{\mathrm{k}} \xi_{\mathrm{j}-\mathrm{k}}
$$

The orbit of $\xi$ is generic with respect to the basis $\left\{X_{1}^{*}, \ldots, X_{n}^{*}\right\}$ is and only if $\xi_{1} \neq 0$, and the jumping indices are 2 to $n$. The cross section $X_{\xi_{1}}$ for the set of generic orbit is given by, $X_{\xi_{1}}=\left\{\xi=\left(\xi_{1}, 0, \xi_{3}, \ldots, \xi_{\mathrm{n}-1}, 0\right): \xi_{1} \in \mathfrak{R}, \xi_{1} \neq 0\right\}$

For $\xi \in \mathrm{g}_{\mathrm{n}}^{*}$, let $\pi_{\xi}$ denote the irreducible representation of $\mathrm{G}_{\mathrm{n}}$, absociated with $\xi$. Then the mapping $\xi \rightarrow \pi_{\xi}$ is bijection of $X_{\xi}$ and the set of all generic irredicible representation. Plancherel measure on $\hat{\mathrm{G}}_{\mathrm{n}}$ is supported by these $\pi_{\xi}$. Denoting by $\mathrm{F}$ the fourier transform on $\mathrm{R}^{\mathrm{n}-1}$, it follows that the Hilbert Schmidt norm of the operator. $\pi_{\xi}(\mathrm{f}), \in \mathrm{L}^{1} \cap \mathrm{L}^{2}\left(\mathrm{G}_{\mathrm{n}}\right)$ is given by

$$
\left\|\pi_{\xi}(\mathrm{f})\right\|_{\mathrm{HS}}^{2}=\int_{\mathrm{R}^{2}} \mathrm{Ff}\left\{\mathrm{p}_{1}(\xi, \mathrm{t}), \ldots, \mathrm{P}_{\mathrm{n}-1}(\xi, \mathrm{t}), \mathrm{t}-\mathrm{s}\right\}^{2} \mathrm{ds} \mathrm{dt}
$$

Theorem 2.1 (Hardy) Suppose $\mathrm{f}$ is measurable function on $\mathfrak{R}$ such that

$$
|\mathrm{f}(\mathrm{x})| \leq \mathrm{C} \mathrm{e}^{-\alpha \mathrm{x}^{2}}, \hat{\mathrm{f}}(\xi) \leq \mathrm{C} \mathrm{e}^{-\square \xi^{2}}, \mathrm{x}, \xi \in \mathfrak{R}
$$

where $\alpha, \beta$ and $\mathrm{C}$ are positive constant. If $\alpha \beta>\frac{1}{4}$ then $\mathrm{f}=0$ a.e.

If $\alpha \beta<\frac{1}{4}$ there are infinitely many linearly independent functions satisfying (1.1), if $\alpha \beta=\frac{1}{4}$ then $\mathrm{f}(\mathrm{x})=\mathrm{C} \mathrm{e}^{-\alpha \mathrm{x}^{2}}$. More precisely, let the fourier transform be defined by

$$
\hat{\mathrm{f}}(\mathrm{y})=\int_{\mathfrak{R}} \mathrm{f}(\mathrm{x}) \exp (-2 \pi \mathrm{x} y) \mathrm{dx}, \mathrm{y} \in \mathfrak{R}
$$

For a proof the above theorem see [6], theorem 3.2 Hardy's theorem is also valid in $\mathfrak{R}^{\mathrm{n}}$ (see [10] for a proof). A generalization of Hardy's theorem due to cowling and 
price asserts that if $\mathrm{a}, \mathrm{b}$ are non-negative constants such that $\mathrm{ab} \geq \frac{1}{4}$, then the only $\mathrm{f} \in \mathrm{S}^{\prime}$ satisfying $\left\|\mathrm{e}^{\mathrm{ax}} \mathrm{f}\right\|_{\mathrm{p}}+\left\|\mathrm{e}^{\mathrm{by}} \hat{\mathrm{f}}\right\|_{\mathrm{q}}<\infty$ for $1 \leq \mathrm{p}, \mathrm{q} \leq \infty$ with at least one of them finite is $\mathrm{f}=0$. On the other hand, if $\mathrm{ab}<\frac{1}{4}$, there are infinitely many $\mathrm{f} \in \mathrm{S}$ satisfying $\left\|\mathrm{e}^{\mathrm{ax}} \mathrm{f}\right\|_{\mathrm{p}}+\left\|\mathrm{e}^{\mathrm{by}^{2}} \hat{\mathrm{f}}\right\|_{\mathrm{q}}<\infty$ (see [2]). Another theorem of this kind is due to A Beurling which says that if $\mathrm{f} \in \mathrm{L}^{1}(\mathfrak{R})$ is such that

$\int_{\mathfrak{R}^{2}} \int|\mathrm{f}(\mathrm{x}) \| \hat{\mathrm{f}}(\mathrm{y})| \mathrm{e}^{|\mathrm{xy}|} \mathrm{dx} d \mathrm{y}<\infty$ then $\mathrm{f}=0$ a.e. one can see that Hardy's theorem can be deduced from this more general theorem of Beurling. This class of results can also be viewed as some sort of uncertainty principle. For an elaboration of this point of view see [10] and the bibliographies in this paper.

\subsection{Definition (Lie Groups)}

Let $\mathrm{G}$ be a topological group. Suppose there is an analytic structure on the set $\mathrm{G}$, compatible with its topology, which converts it into an analytic manifold and for which the maps

$$
\begin{array}{ll}
(x, y) \rightarrow x y & x, y \in G \\
x \rightarrow x^{-1} & x \in G
\end{array}
$$

of $\mathrm{G} \times \mathrm{G}$ into $\mathrm{G}$ is and of $\mathrm{G}$ into $\mathrm{G}$, respectively, are both analytic. Then, $\mathrm{G}$ together with this analytic structure, is called a Lie group.

Example: $\mathfrak{R}^{\mathrm{m}}$, the additive group of m-tuples of real numbers is a real analytic group. $\mathbf{C}^{\mathrm{m}}$, the additive group of $\mathrm{m}$-tuples of complex numbers, is a complex analytic group.

2.3 Nilpotent Lie Algebras: Let $g$ be a Liealgebra over $\mathfrak{R}$. We say that $g$ is a nilpotent Lie algebra if there is an integer $n$ such that $g^{(n+1)}=(0)$. If $g^{(n)} \neq(0)$ as well, so that $\mathrm{n}$ is minimal, then $\mathrm{g}$ is said to be $\mathrm{n}$-step nilpotent.

2.4 Nilpotent Lie Groups: A nilpotent Lie group G is one whose Lie algebra $\mathrm{g}$ is nilpotent.

$\mathrm{G}_{4}$ is a group of higher dimension whose underlying set is $\mathfrak{R}^{4}$. The multiplication and inverse of elements of $\mathrm{G}_{4}$ is defined by,

$$
\left.\left(\mathrm{x}_{1}, \ldots, \mathrm{x}_{4}\right)\left(\mathrm{y}_{1}, \ldots, \mathrm{y}_{4}\right)=\mathrm{x}_{1}+\mathrm{y}_{1}+\mathrm{x}_{4} \mathrm{y}_{2}+\frac{1}{2} \mathrm{x}_{4}^{2} \mathrm{y}_{3}, \mathrm{x}_{2}+\mathrm{y}_{2}+\mathrm{x}_{4} \mathrm{y}_{3}, \mathrm{x}_{3}+\mathrm{y}_{3}, \mathrm{x}_{4}+\mathrm{y}_{4}\right)
$$

and

$$
\left(\mathrm{x}_{1}, \ldots, \mathrm{x}_{4}\right)^{-1}=\left(-\mathrm{x}_{1}+\mathrm{x}_{2} \mathrm{x}_{4}-\frac{1}{2} \mathrm{x}_{3} \mathrm{x}_{4}^{2},-\mathrm{x}_{2}+\mathrm{x}_{3} \mathrm{x}_{4},-\mathrm{x}_{3},-\mathrm{x}_{4}\right)
$$

Theorem 2.5 Let $\mathrm{f} \in \mathrm{L}^{1}\left(\mathrm{G}_{4}\right) \cap \mathrm{L}^{2}\left(\mathrm{G}_{4}\right)$ satisfies the following $(\alpha)$

$$
\int_{G_{4}} e^{p a \pi\|x\|^{2}}|f(x)|^{p} d x<\infty
$$


( $\beta) \quad \int_{\Re^{2}} \mathrm{e}^{\mathrm{b} \pi \mathrm{q}\left(\xi_{1}{ }^{2}+\xi_{3}{ }^{2}\right)}\left|\xi_{1}\right|\left\|\pi_{\xi_{1}, \xi_{3}}(\mathrm{f})\right\|_{\text {HS }}^{\mathrm{q}} \mathrm{d} \xi_{1}, \mathrm{~d} \xi_{3}<\infty$

If $\mathrm{p}<\infty$ then

(i) for $q \geq 2$ and $a b>1$, we have $f=0$ a.e.

(ii) for $1 \leq \mathrm{q}<2$ and $\mathrm{ab}>2$, we have $\mathrm{f}=0$ a.e.

Proof: The proof is a reduction to the case $\mathrm{p}=\infty$.

$$
\begin{aligned}
& \left\|\left(\mathrm{u}_{1}, \mathrm{u}_{2}, \mathrm{u}_{3}, \mathrm{u}_{4}\right)^{-1}\left(\mathrm{x}_{1}, \mathrm{x}_{2}, \mathrm{x}_{3}, \mathrm{x}_{4}\right)\right\| \\
= & \left\|\left(-\mathrm{u}_{1}, \mathrm{u}_{2} \mathrm{u}_{4}-\frac{1}{2} \mathrm{u}_{3} \mathrm{u}_{4}^{2},-\mathrm{u}_{2}+\mathrm{u}_{3} \mathrm{u}_{4},-\mathrm{u}_{3},-\mathrm{u}_{4}\right)\left(\mathrm{x}_{1}, \mathrm{x}_{2}, \mathrm{x}_{3}, \mathrm{x}_{4}\right)\right\| \\
= & \left\|\left(\mathrm{x}_{1}-\mathrm{u}_{1}+\mathrm{u}_{2} \mathrm{u}_{4}-\frac{1}{2} \mathrm{u}_{3} \mathrm{u}_{4}^{2}-\mathrm{u}_{4} \mathrm{x}_{2}+\frac{1}{2} \mathrm{u}_{4}^{2} \mathrm{x}_{3}, \mathrm{x}_{2}-\mathrm{u}_{2}+\mathrm{u}_{3} \mathrm{u}_{4}-\mathrm{u}_{4} \mathrm{x}_{3}, \mathrm{x}_{3}-\mathrm{u}_{3}, \mathrm{x}_{4}-\mathrm{u}_{4}\right)\right\| \\
= & \left\|\left(\mathrm{x}_{1}-\mathrm{u}_{1}-\mathrm{u}_{4}\left(\mathrm{x}_{2}-\mathrm{u}_{2}\right)-\frac{1}{2} \mathrm{u}_{4}^{2}\left(\mathrm{u}_{3}-\mathrm{x}_{3}\right), \mathrm{x}_{2}-\mathrm{u}_{2}-\mathrm{u}_{4}\left(\mathrm{x}_{3}-\mathrm{u}_{3}\right), \mathrm{x}_{3}-\mathrm{u}_{3}, \mathrm{x}_{4}-\mathrm{u}_{4}\right)\right\| \\
\geq \quad & \left\|\left(\mathrm{x}_{1}, \mathrm{x}_{2}, \mathrm{x}_{3}, \mathrm{x}_{4}\right)\right\|-\left\|\left(\mathrm{u}_{1}, \mathrm{u}_{2}, \mathrm{u}_{3}, \mathrm{u}_{4}\right)\right\|-\left\|\left(\mathrm{u}_{4}\left(\mathrm{x}_{2}-\mathrm{u}_{2}\right), 0,0,0\right)\right\|-\|\left(\frac{1}{2} \mathrm{u}_{4}^{2}\left(\mathrm{u}_{3}-\mathrm{x}_{3}\right),\right. \\
= & \left.\mathrm{u}_{4}\left(\mathrm{x}_{3}-\mathrm{u}_{3}\right), 0,0\right) \| \\
= & \left\|\left(\mathrm{x}_{1}, \mathrm{x}_{2}, \mathrm{x}_{3}, \mathrm{x}_{4}\right)\right\|-\left\|\left(\mathrm{u}_{1}, \mathrm{u}_{2}, \mathrm{u}_{3}, \mathrm{u}_{4}\right)\right\|-\left|\mathrm{u}_{4}\right|\left|\mathrm{x}_{2}-\mathrm{u}_{2}\right|-\left|\mathrm{u}_{4}-\mathrm{x}_{3}\right|\left|\mathrm{u}_{4}\right| \sqrt{\frac{1}{4} \mathrm{u}_{4}^{2}+1} \\
& \left(\mathrm{u}_{1}, \mathrm{u}_{2}, \mathrm{u}_{3}, \mathrm{u}_{4}\right)=\left(\mathrm{x}_{1}, \mathrm{x}_{2}, \mathrm{x}_{3}, \mathrm{x}_{4}\right)
\end{aligned}
$$

For $\mathrm{u} \in\left\{\mathrm{u}:\|\mathrm{u}\| \leq \frac{1}{\mathrm{~m}}\right\}$ and $\mathrm{x} \in \mathrm{G}_{4}$ s.t. $\|\mathrm{x}\|>1$, we have

$$
\left\|\mathrm{u}^{-1} \mathrm{x}\right\| \geq\|\mathrm{x}\|-\frac{1}{\mathrm{~m}}-\|\mathrm{u}\|\left(\left|\mathrm{x}_{2}\right|+\left|\mathrm{u}_{2}\right|\right)-\left(\left|\mathrm{u}_{3}\right|+\left|\mathrm{x}_{3}\right|\right)\left|\mathrm{u}_{4}\right| \sqrt{\frac{1}{4} \mathrm{u}_{4}^{2}+1}
$$

$\geq \quad\|\mathrm{x}\|-\frac{1}{\mathrm{~m}}-\|\mathrm{u}\|(\|\mathrm{x}\|+\|\mathrm{u}\|)-(\|\mathrm{u}\|+\|\mathrm{x}\|)\|\mathrm{u}\|\left(1+\frac{1}{2}\left|\mathrm{u}_{4}\right|\right)$

$\geq \quad\|x\|-\frac{1}{m}-\frac{1}{m}\left(\|x\|+\frac{1}{m}\right)-\left(\frac{1}{m}+\|x\|\right) \frac{1}{m}\left(1+\frac{1}{2 m}\right)$

$=\quad\|x\|-\frac{1}{m}-\frac{1}{m^{2}}-\frac{1}{m^{2}}\left(1+\frac{1}{2 m}\right)-\|x\|\left(\frac{1}{m}+\frac{1}{m}+\frac{1}{2 m^{2}}\right)$

$=\quad\|\mathrm{x}\|-\frac{1}{\mathrm{~m}}-\frac{2}{\mathrm{~m}^{2}}-\frac{1}{2 \mathrm{~m}^{3}}-\|\mathrm{x}\|\left(\frac{2}{\mathrm{~m}}+\frac{1}{2} \mathrm{~m}^{2}\right)$

$\geq \quad\|\mathrm{x}\|\left(1-\frac{1}{\mathrm{~m}}-\frac{2}{\mathrm{~m}^{2}}-\frac{1}{2 \mathrm{~m}^{3}}-\frac{2}{\mathrm{~m}}-\frac{1}{2 \mathrm{~m}^{2}}\right) \quad($ since $\|\mathrm{x}\|>1)$

$=\quad\|x\|\left(1-\frac{3}{m}-\frac{5}{2 m^{2}}-\frac{1}{2 m^{3}}\right)$

Let $\mathrm{g}$ be a continuous function with compact support with supp $\mathrm{g} \subset\left\{\mathrm{u}=\left(\mathrm{u}_{1}, \mathrm{u}_{2}, \mathrm{u}_{3}\right.\right.$, $\left.\left.\mathrm{u}_{4}\right):\|\mathrm{u}\| \leq \frac{1}{\mathrm{~m}}\right\}$.

Let $\mathrm{x}=\left(\mathrm{x}_{1}, \mathrm{x}_{2}, \mathrm{x}_{3}, \mathrm{x}_{4}\right) \in \mathrm{G}_{4}$ be s.t. $\|\mathrm{x}\|>1$. Then $\left\|\mathrm{u}^{-1} \mathrm{x}\right\| \geq\|\mathrm{x}\|\left(1-\frac{3}{\mathrm{~m}}-\frac{5}{2 \mathrm{~m}^{2}}-\frac{1}{2 \mathrm{~m}^{3}}\right)$ 
for all $\mathrm{u} \in \operatorname{supp} \mathrm{g}$.

Denote $\mathrm{e}_{\mathrm{a}}(\mathrm{x})=\mathrm{e}^{\mathrm{a} \pi\|\mathrm{x}\|^{2}}$

by $(\alpha) \mathrm{e}_{\mathrm{a}}|\mathrm{f}| \in \mathrm{L}^{\mathrm{p}}\left(\mathrm{G}_{4}\right)$ so $|\mathrm{g}| * \mathrm{e}_{\mathrm{a}}|\mathrm{f}| \in \mathrm{L}^{\infty}\left(\mathrm{G}_{4}\right)$

Let $\mathrm{C}=\left\||\mathrm{g}| * \mathrm{e}_{\mathrm{a}}|\mathrm{f}|\right\|_{\infty}$ and let $\|\mathrm{x}\|>1$,

$\mathrm{C} \quad \geq\left\|\mathrm{g}\left|* \mathrm{e}_{\mathrm{a}}\right| \mathrm{f}\right\|(\mathrm{x})$

$=\int|g|(u) e_{a}|f|\left(u^{-1} x\right) d u$

$\left.=\int|g|(u) e_{a}\left(u^{-1} x\right)|f| \mid u^{-1} x\right) d u$

$=\int|g|(u) e^{a \pi\left\|u^{-1} x\right\|^{2}}|f|\left(u^{-1} x\right) d u$

$\left.\geq \quad \int|g|(u) e^{a \pi\|x\|^{2}\left(1-3 / m-5 / m^{2}-1 / 2 m^{3}\right)}|f| \mid u^{-1} x\right) d u$

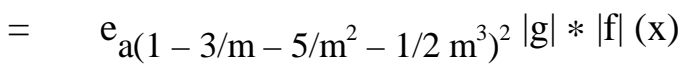

Hence for $\mathrm{x} \in \mathrm{G}_{4}$ with $\|\mathrm{x}\|>1$

$$
|\mathrm{g} * \mathrm{f}(\mathrm{x})| \leq|\mathrm{g}| *|\mathrm{f}|(\mathrm{x}) \leq \mathrm{C} \mathrm{e}^{-\pi \mathrm{a}\left(1-3 / \mathrm{m}-5 / \mathrm{m}^{2}-1 / 2 \mathrm{~m}^{3}\right)^{2}\|\mathrm{x}\|^{2}}
$$

Since $\mathrm{g} * \mathrm{f}$ is continuous (or $\{\mathrm{x}:\|\mathrm{x}\| \leq 1\}$ is a compact set)

We have,

$$
|\mathrm{g} * \mathrm{f}(\mathrm{x})| \leq \text { const } \mathrm{e}^{-\pi \mathrm{a}\left(1-3 / \mathrm{m}-5 / \mathrm{m}^{2}-1 / 2 \mathrm{~m}^{3}\right)^{2}\|\mathrm{x}\|^{2}}
$$

for all $\mathrm{x} \in \mathrm{G}_{4}$

Also

$\left\|\pi_{\xi_{1}, \xi_{3}}(\mathrm{~g} * \mathrm{f})\right\|_{\mathrm{HS}} \leq\left\|\pi_{\xi_{1}, \xi_{3}}(\mathrm{~g})\right\|_{\mathrm{op}}\left\|\pi_{\xi_{1}, \xi_{3}}(\mathrm{f})\right\|_{\mathrm{HS}}$

( \|\|$_{\text {op }}$ is the operator norm)

$\leq\|\mathrm{g}\|_{1}\left\|\pi_{\xi_{1}, \xi_{3}}(\mathrm{f})\right\|_{\mathrm{HS}}$

So $\quad \int_{\Re^{2}} \mathrm{e}^{\mathrm{b} \pi \mathrm{q}\left(\xi_{1}{ }^{2}+\xi_{3}{ }^{2}\right)}\left|\xi_{1}\right| \| \pi_{\xi_{1}, \xi_{3}}\left(\mathrm{~g} * \mathrm{f} \|_{\mathrm{HS}}^{\mathrm{q}} \mathrm{d} \xi_{1} \mathrm{~d} \xi_{3}\right.$

$$
\begin{aligned}
& \leq\|g\|_{1}^{\mathrm{q}} \int_{\Re^{2}} \mathrm{e}^{\mathrm{b} \pi \mathrm{q}\left(\xi_{1}^{2}+\xi_{3}^{2}\right)}\left|\xi_{1}\right|\left\|\pi_{\xi_{1}, \xi_{3}}(\mathrm{f})\right\|_{\mathrm{HS}}^{\mathrm{q}} \mathrm{d} \xi_{1} \mathrm{~d} \xi_{3} \\
& <\infty
\end{aligned}
$$

Choosing $\mathrm{m}$ sufficiently large so that

$$
\text { ab }\left(1-3 / m-5 / m^{2}-1 / 2 m^{3}\right)^{2}>1 \quad(\text { or }>2)
$$

We have,

$\mathrm{g} * \mathrm{f}=0$ ae by the previous case. Choosing $\mathrm{g}$ to be approximate identity we get $\mathrm{f}=0$ a.e.

Theorem 2.6 Let $\mathrm{f}: \mathrm{G}_{4} \rightarrow \mathrm{C}$ be measurable and

(a) $\left|f\left(x_{1}, x_{2}, x_{3}, x_{4}\right)\right| \leq C g\left(x_{2}, x_{3}, x_{4}\right) e^{-a \pi\left|x_{1}\right|^{p}}$

(b) $\left\|\pi_{\xi_{1}, \xi_{3}}(\mathrm{f})\right\|_{\mathrm{HS}} \leq \mathrm{C} \mathrm{e}^{-\mathrm{b} \pi\left(\left|\xi_{1}\right|^{\mathrm{q}}+\left|\xi_{3}\right|^{\mathrm{q}}\right)}$ 
where a, b, C > $0, g \in \mathrm{L}^{1}\left(\mathfrak{R}^{3}\right) \cap \mathrm{L}^{2}\left(\mathfrak{R}^{3}\right), \mathrm{p} \geq 1, \frac{1}{\mathrm{p}}+\frac{1}{\mathrm{q}}=1$

If $(\mathrm{ap})^{1 / \mathrm{p}}(\mathrm{bq})^{1 / \mathrm{q}}>2$ then $\mathrm{f}=0$ a.e.

Proof: Let $\mathrm{V}$ be as in earlier pages then

$$
\begin{aligned}
& \left|\mathrm{V}\left(\mathrm{x}_{1}\right)\right| \leq \int_{\mathfrak{R}^{4}}\left|\mathrm{f}\left(\mathrm{y}_{1}, \mathrm{x}_{2}, \mathrm{x}_{3}, \mathrm{x}_{4}\right)\right|\left|\mathrm{f}\left(\mathrm{y}_{1}-\mathrm{x}_{1}, \mathrm{x}_{2}, \mathrm{x}_{3}, \mathrm{x}_{4}\right)\right| d \mathrm{dx}_{2} \mathrm{dx}_{3} \mathrm{dx} d \mathrm{y}_{1} \\
& \leq \quad \mathrm{C} \int_{\Re^{4}}\left(\mathrm{~g}\left(\mathrm{x}_{2}, \mathrm{x}_{3}, \mathrm{x}_{4}\right)\right)^{2} \mathrm{e}^{-\mathrm{a} \pi\left(\mathrm{y}_{1}{ }^{\mathrm{p}}+\left(\mathrm{y}_{1}-\mathrm{x}_{1}\right)^{\mathrm{p}}\right)} \\
& \leq \quad \mathrm{C}\|\mathrm{g}\|_{2}^{2} \int_{\Re} \mathrm{e}^{-\mathrm{a} \pi\left(\mathrm{y}_{1}^{\mathrm{p}}+\left(\mathrm{y}_{1}-\mathrm{x}_{1}\right)^{\mathrm{p}}\right)} \mathrm{dy_{1 }} \\
& \mathrm{y}_{1}^{\mathrm{p}}+\left(\mathrm{y}_{1}-\mathrm{x}_{1}\right)^{\mathrm{p}}=\left(\mathrm{y}_{1}{ }^{2}\right)^{\mathrm{p} / \mathrm{q}}+\left(\left(\mathrm{y}_{1}-\mathrm{x}_{1}\right)^{2}\right)^{\mathrm{p} / 2} \\
& \geq \quad 2^{1-p / 2}\left(y_{1}^{2}+\left(y_{1}-x_{1}\right)^{2}\right)^{p / 2} \quad \begin{array}{ll}
\text { for } a, b \geq 0 \\
a^{p}+b^{p} \geq 2^{1-p}(a+b)^{p}
\end{array} \\
& =2^{1-\mathrm{p} / 2}\left[\frac{1}{2}\left[\left(2 \mathrm{y}_{1}-\mathrm{x}_{1}\right)^{2}+\mathrm{x}_{1}{ }^{2}\right]\right]^{\mathrm{p} / 2} \\
& =\quad 2^{1-\mathrm{p}}\left[\mathrm{x}_{1}{ }^{2}+\left(2 \mathrm{y}_{1}-\mathrm{x}_{1}\right)^{2}\right]^{\mathrm{p} / 2} \geq 2^{1-\mathrm{p}}\left[\left|\mathrm{x}_{1}{ }^{\mathrm{p}}\right|+\left|2 \mathrm{y}_{1}-\mathrm{x}_{1}^{\mathrm{p}}\right|\right]
\end{aligned}
$$

Hence, $\left|\mathrm{V}\left(\mathrm{x}_{1}\right)\right| \leq \mathrm{C}\|\mathrm{g}\|_{2}^{2} \mathrm{e}^{-\mathrm{a} \pi 2^{1-\mathrm{p}}\left|\mathrm{x}_{1}\right|^{\mathrm{p}}} \int_{\Re} \mathrm{e}^{-\mathrm{a} \pi\left|2 \mathrm{y}_{1}-\mathrm{x}_{1}\right|^{p}} \mathrm{dy_{1 }}$

$$
\begin{gathered}
\leq \text { const } \mathrm{e}^{-\mathrm{a} \pi 2^{1-\mathrm{p}}\left|\mathrm{x}_{1}\right|^{\mathrm{p}}} \\
\left|\hat{\mathrm{V}}\left(\xi_{1}\right)\right|=\left|\xi_{1}\right| \int_{\Re}\left\|\pi_{\xi_{1}, \xi_{3}}(\mathrm{f})\right\|_{\mathrm{HS}}^{2} \mathrm{~d} \xi_{3} \\
\leq \mid \xi_{1} \int_{\Re} \mathrm{e}^{-2 \mathrm{~b} \pi\left(\left|\xi_{1}\right|^{\mathrm{q}}+\left|\xi_{3}\right|^{\mathrm{q}}\right)} \mathrm{d} \xi_{3} \\
\leq \text { const. }\left|\xi_{1}\right| \mathrm{e}^{-2 \mathrm{~b} \pi}\left|\xi_{1}\right|^{\mathrm{q}}
\end{gathered}
$$

Choose b' $<$ b s.t. $(\text { ap })^{1 / p}\left(b^{\prime} q\right)^{1 / q}>2$, we have

$$
\begin{aligned}
& \left|\hat{\mathrm{V}}\left(\xi_{1}\right)\right| \leq \text { const } \mathrm{e}^{-2 \mathrm{~b}^{\prime} \pi\left|\xi_{1}\right|^{q}} \\
& \left(\mathrm{a} 2^{1-\mathrm{p}} \mathrm{p}\right)^{1 / \mathrm{p}}\left(2 \mathrm{~b}^{\prime} \mathrm{q}\right)^{1 / \mathrm{q}}=(\mathrm{ap})^{1 / \mathrm{p}}\left(\mathrm{b}^{\prime} \mathrm{q}\right)^{1 / \mathrm{q}} 2^{(1-\mathrm{p}) / \mathrm{p}+1 / \mathrm{q}}>2
\end{aligned}
$$

So $\mathrm{V}=0$ a.e. Hence $\mathrm{f}=0$ a.e.

\section{REFERENCES}

1. Bhatta, C.R., 2009, Hardy Uncertainty Principle for Low Dimensional Nilpotent Liegroup $\mathrm{G}_{4}$. Nepal Journal of Science and Technology 10, 155 - 159.

2. Cowling M. Price J. 1983. Generalisation of Heisenberg's inequalities Harmonic analysis, Proceeding Corotona Italy (1982). Maueeri G. Ricci F and Weiss G. Eds. Lecture notes No. 982. Springer Verlag.

3. Cowling M. G., Sitaram A. Sundari M. 2000. Hardy Uncertainty Principle on semi-simple Liegroups, Pacific J. Math 192, 293 - 296. 
4. Dym H and Mckean H. P. 1972. Fourier series and integrals. Academic Press. New York.

5. Folland G.B., Sitaram, A., 1997. The Uncertainty Principle: A mathematical Survey, J. Fourier analysis and application 3, 207-238.

6. Hardy G. H., 1933. A theorem concerning Fourier transforms, J. London Math Soc, 8, 227-231.

7. Hewitt, E. and Ross, K.A., (1963, 1970). Abstract Harmonic analysis I and II Springer Verlag.

8. Ole, A. Nelson, (1983), Unitary representations and coadjoint orbits of low dimensional nilpotent liegroups, queens paper in pure and applied mathematics, no. 63.

9. Reiter, H. and Stegemen, J.D. 2000. Classical Harmonic analysis and locally compact groups. Oxford University press.

10. Sitaram A, Sundari, M \& Thangavelu, S. 1995. Uncertainty Principle on Certain Lie groups. Proc. Indian Acad. of Science 105, No. 2, 135-151. 